\title{
Adenocarcinoma Ex Goblet Cell Carcinoid (GCC) of the Appendix: Report of Five Cases and Pitfalls in Diagnosis of GCC
}

Jinhua Piao ${ }^{1}$, Jula Veerapong ${ }^{2}$, Zhenyan $\mathrm{Li}^{3}$, Edward Bolesta ${ }^{1}$, Nishant Poddar ${ }^{4}$ and Jin-Ping Lai ${ }^{{ }^{*}}$

${ }^{1}$ Department of Pathology, Saint Louis University School of Medicine, Saint Louis, MO 63104, USA

${ }^{2}$ Department of Surgery, Saint Louis University School of Medicine, Saint Louis, MO 63104, USA

${ }^{3}$ Division of Oncology, Washington University School of Medicine, Saint Louis, MO 63110, USA

${ }^{4}$ Division of Hematology-Oncology, Saint Louis University School of Medicine, Saint Louis, MO 63104, USA

"Corresponding author: Jinping Lai, MD, PhD, Department of Pathology, Saint Louis University, 1402 South Grand Blvd, St. Louis, MO 63104, USA, Tel: 314-577-8475; Fax: 314-577-6132; E-mail: jinpinglai@slu.edu

Received date: Dec 02, 2015; Accepted date: Jan 08, 2016; Published date: Jan 14, 2016

Copyright: ( 2016 Piao J, et al. This is an open-access article distributed under the terms of the Creative Commons Attribution License, which permits unrestricted use, distribution, and reproduction in any medium, provided the original author and source are credited.

\begin{abstract}
Neoplasm of the appendix is relatively rare. Only $0.9-1.4 \%$ of all appendectomy specimens is found to have it. One in particular is the adenocarcinoma ex goblet cell carcinoid. The exact histopathogenesis and pathologic classification of this neoplasm are yet to be elucidated. Herein we report five cases to emphasize the importance of meticulous sampling and the possibility of misdiagnosis due to the presence of diverticulitis and acute appendicitis in some of these patients. All of our patients initially were presented with symptoms of or mimicking appendicitis, with radiology imaging suggestive of acute appendicitis or an appendiceal abscess. The pathologic examination of the appendectomy specimen revealed the incidental finding of the adenocarcinoma ex goblet cell carcinoid with focal positivity of synaptophysin and chromogranin. Two of our patients had diverticulitis and perforated appendicitis, which may lead to a misdiagnosis of the goblet cell carcinoid due to the absence of a discrete mass formation and focal localization of these tumor cells. Therefore, meticulous sampling is imperative in the diagnosis of this entity.
\end{abstract}

Keywords: Neoplasm; Adenocarcinoma; Interventional radiology; Streptococcus angiosus, Laparoscopic; Appendicitis

\section{Introduction}

Neoplasm of the appendix is relatively rare, with only $0.9-1.4 \%$ of all appendectomy specimens found to have it [1,2]. The GCC was first described in 1974 as a separate entity [3], and counts for less than $5 \%$ of all primary appendix neoplasms, with an average age of 58.8 [4]. Adenocarcinoma ex goblet cell carcinoid is an uncommon entity characterized by both neuroendocrine differentiation and adenocarcinoma of the colon. Its histogenesis and pathogenesis still remain controversial [5-9], although it has been suggested that GCC cells are from lysozyme-producing cells in small intestinal crypts [10]. Initially, GCC was believed to be a low-grade malignancy [3], however, later studies suggested higher malignant potentials of the GCC compared to the classic appendix carcinoids [11]. In this study, we reported five new cases with symptoms of or mimicking appendicitis, and radiology imaging findings suggestive of appendicitis or an appendiceal abscess. We reviewed the literature and discussed the importance of meticulous sampling and the pitfalls of misdiagnosis due to the presence of diverticulitis in some of these patients.

\section{Case Reports}

\section{Case 1}

A 60- year-old male presented to the Emergency Department for an abdominal pain and was found to have perforated appendicitis with an abscess on the CT scan of the abdomen. The interventional radiology (IR) drain revealed purulent fluid, and the patient was treated with a two-week course of Ciprofloxacin and Flagyl, and was later discharged. Two months later, the patient was presented with a persistent loculated right lower quadrant (RLQ) fluid, and the drain study showed the fluid density concerning for appendiceal mucocele instead of the previously thought abscess.

One month later, the patient was presented with a localized, sharp RLQ pain and a dark red fluid draining from his RLQ drain, as well as associated symptoms of hot flashes and dizziness. The CT scan of the abdomen and pelvis was suggestive of appendiceal mucinous neoplasm, so the patient was admitted for further treatments. Past medical history was significant for gastroesophageal reflux disease (GERD), gout, chronic back pain, depression, and tinea versicolor of the chest. Surgical history was significant for inguinal hernia repair, and family history was significant for breast cancer in the patient's mother and sister.

Physical examination revealed serous drainage from the RLQ drain and the abdomen was soft and non-distended, with no tenderness to palpation. On admission, the patient weighed $173 \mathrm{lbs}(78.5 \mathrm{~kg})$, and his height was 5'7" (1.7 m) with a body mass index (BMI) of $27.1 \mathrm{~kg} / \mathrm{m}^{2}$. Initial laboratory studies revealed unremarkable results for complete blood count (CBC), basic metabolic panel (BMP), carcinoembryonic antigen (CEA) $(0.9 \mathrm{ng} / \mathrm{ml})$, carbohydrate antigen CA19-9 $(6 \mathrm{U} / \mathrm{ml})$, and cancer antigen CA125 $(10.6 \mathrm{U} / \mathrm{ml})$. The CT of the abdomen and pelvis revealed abnormal tubular fluid collection in the RLQ measuring $3.7 \times 3.8 \times 7.0 \mathrm{~cm}$ (Figure 1A). The CT-guided drainage aspirated $25 \mathrm{ml}$ of mucus fluid from the RLQ. On further workup, the cytopathology of this fluid revealed mucin and chronic inflammation, and showed no evidence of malignancy. Cultures showed negative growth for anaerobes, fungus, and acid-fast organisms, except positive growth for Streptococcus angiosus after 48 hours. 


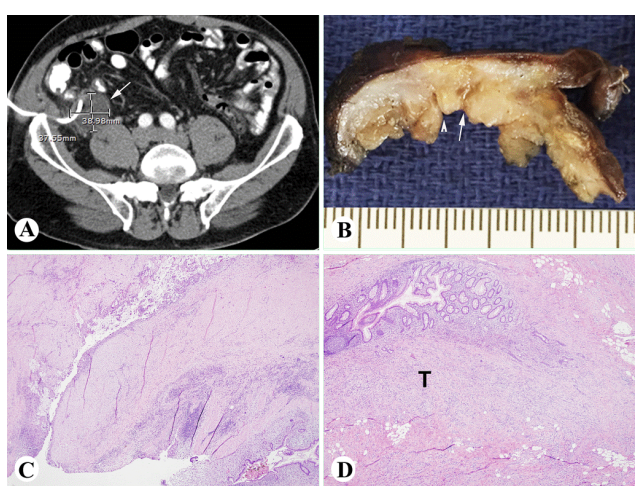

Figure 1: A CT scan of the abdomen and pelvis shows tubular fluid collection in the RLQ $(3.7 \times 3.8 \times 7.0 \mathrm{~cm})$ with a drainage catheter in its superior aspect (arrow); B: Grossly, multiple diverticula (arrowhead and arrow) extending into the muscularis layer causing fistula and abscess formation (arrow); C: H\&E stain showing fistula formation (40x); D: H\&E stain showing tumor cells composed of goblet cells and singlet signet-ring cells $(\mathrm{T})$ infiltrating the appendiceal wall (40x).

The patient underwent laparoscopic appendectomy and the specimen was obtained for histopathological examination. Grossly, the appendix had an increased wall thickness ranging from $0.5 \mathrm{~cm}$ to 1.5 $\mathrm{cm}$ with a pink-tan mucosal surface and prominent mucosal folds. The appendiceal lumen was filled with mucin and purulent fluid. Multiple inflammatory/pseudopolyps were identified on the surface of the mucosa ranging from $0.3 \times 0.3 \times 0.3 \mathrm{~cm}$ to $0.5 \times 0.5 \times 0.4 \mathrm{~cm}$. Multiple diverticula were also identified, grossly extending into the muscularis layer (Figure 1B).

Hematoxylin and Eosin (H\&E) stains revealed multiple diverticula and fistula formations (Figure 1C), and tumor cells composed of goblet cell carcinoids and signet-ring cell carcinoma (Figures 1D, 2A-2B) infiltrating the appendiceal wall, predominantly located at the base (orifice) of the appendix which focally involved the proximal resection margin. Immunohistochemical stains were performed with adequate controls.

The tumor cells were focally positive for synaptophysin (Figure 2C) and chromogranin (Figure 2D, left), diffusely positive for CK20, CDX2 (Figure 2D, right) and CEA, and negative for CK7. The Ki-67/MIB-1 stain highlighted scattered tumor nuclei of the goblet cell carcinoids with more prominent staining in the singlet signet ring cells. The tumor was diagnosed as adenocarcinoma ex goblet cell carcinoids, signet ring cell type (T3NxMx). However, no tumor was identified in the areas of diverticulitis or previously perforated site.

The patient was later discharged after an uneventful one-day hospital course. The pathology report was then discussed with the patient and he was re-admitted after 15 days from the initial discharge date, for the right hemicolectomy. Rare signet ring tumor cells were present at the prior appendectomy site. The patient has been recovering well from the surgery, and has started on systemic therapy as we report this case.

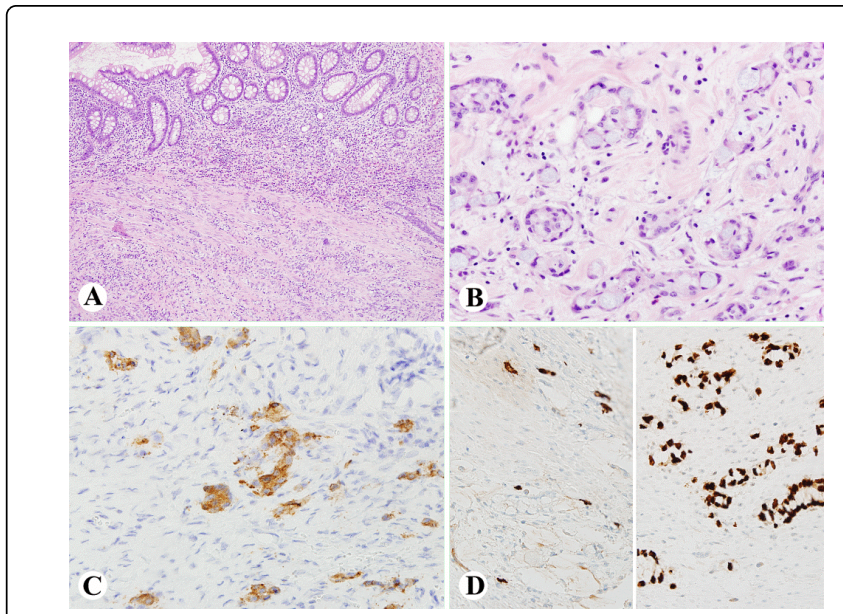

Figure 2: A-B: H\&E staining showing tumor cells composed of goblet cell carcinoids and signet ring cells (A, 100x; B, 400x); C-D: Immunoreactivity of the tumor cells showing focal positive for synaptophysin (C, 400x); and chromogranin (D, left, 400x), diffusely and strongly positive for CDX2 (D, right, 400x).

\section{Case 2}

A 34 year-old male presented with concerning symptoms for appendiceal abscess. Ultrasound (US) showed a large mass surrounding his appendix consistent with abscess. US and CT-guided biopsy was negative for inflammatory or neoplastic process. 14 days later, the repeated CT imaging revealed an enlargement of the mass that was suggestive of a neoplastic process. Subsequently, the patient underwent an exploratory laparotomy which revealed an appendiceal tumor with metastatic disease. The ileocecectomy specimen showed a $7.4 \mathrm{~cm}$ tumor. The $\mathrm{H} \& \mathrm{E}$ stain revealed poorly differentiated adenocarcinoma ex goblet cell carcinoid (T4bN2M1). Lymphovascular invasion and perineural invasion were present, and 14 of 18 lymph nodes were involved by the metastatic tumor. The tumor cells were focally positive for synaptophysin and chromogranin. Intraperitoneal metastasis beyond the RLQ including pseudomyxoma peritonei was present. The patient had a positive response to the chemotherapy with FOLFOX, 5-FU, and Avastin, and showed a $40 \%$ reduction in tumor sizes. The patient is currently considering hyperthermic intraperitoneal chemotherapy (HIPEC) treatment as we report this case.

\section{Case 3}

A 46 year-old male presented with symptoms of RLQ abdominal pain secondary to peritonitis from a perforated appendix. The histopathological examination of the appendectomy specimen revealed a $1.4 \mathrm{~cm}$ Grade 1, well-differentiated goblet cell carcinoid (T2N0Mx). No lymphovascular invasion was present. Subsequently, the patient was informed of his appendectomy specimen pathology report and underwent right hemicolectomy. The right hemicolectomy revealed a $1.2 \mathrm{~cm}$ Grade 1, well-differentiated neuroendocrine tumor of the small intestine. Lymphovascular invasion and perineural invasion were present. The patient was then placed under active surveillance with no evidence of recurrence for the past three years. 


\section{Case 4}

A 65 year-old female presented with symptoms of acute appendicitis, and underwent a non-emergent appendectomy. The appendectomy specimen revealed a greater than $5 \mathrm{~cm}$ poorly differentiated adenocarcinoma ex goblet cell carcinoid, Type C (T3N0M1b). Perineural invasion was present, but no lymphovascular invasion was identified. The proximal margin was positive for carcinoma. The tumor cells were focally positive for chromogranin and synaptophysin. Subsequently, the patient underwent a right hemicolectomy. The right hemicolectomy specimen revealed a residual adenocarcinoma ex goblet cell carcinoid present in the previous appendectomy site. The tumor cells stained positive for CK20 and CAM5.2. Lymphovascular invasion was present. Four years later, the patient developed lower abdominal pain, and the CT of the abdomen/ pelvis revealed a $3.1 \mathrm{~cm}$ ovarian mass. The patient then developed an increased abdominal pain and the follow up ultrasound of the pelvis revealed a $3.9 \times 3.2 \times 2.5 \mathrm{~cm}$ lobulated solid mass in her left adnexa and an adjacent cyst measuring $2.0 \mathrm{~cm}$ in greatest dimension. One month later, the patient underwent laparoscopic bilateral salpingoopherectomy, and the histopathological examination of the tissue revealed metastatic mixed adenoneuroendocrine carcinoma, with a remnant of vaginal cuff involved. The tumor cells stained positive for synaptophysin and chromogranin, and focally positive for CK20. Lymphovascular invasion was present. The patient has been recovering well, and has successfully completed six cycles of FOLFOX, as we report this case.

\section{Case 5}

A 46 year-old male presented with hematochezia, while the CT of his abdomen/pelvis revealed a $3.2 \times 2.7 \times 2.0 \mathrm{~cm}^{3}$ dilated fluid filled appendix. His colonoscopy showed a cecal lesion arising at the appendiceal orifice, and his biopsy revealed an adenocarcinoma ex goblet cell carcinoid. Diverticulosis was also present. The patient continued to have hematochezia and supra-pubic abdominal pain, and subsequently underwent right hemicolectomy. Histopathological examination of the right hemicolectomy specimen revealed a poorly differentiated adenocarcinoma ex goblet cell carcinoid involving the terminal ileum and ascending colon (T4aN2bM1b). The tumor size could not be determined because the tumor does not form a discrete measurable mass, but it was highly infiltrative. Lymphovascular invasion and perineural invasion were present. The tumor cells stained positive for synaptophysin and chromogranin. As we report this case, the patient has been recovering well from the surgery and is currently finishing up his $10^{\text {th }}$ cycle of chemotherapy with FOLFIRI and Avastin.

\section{Discussion}

Here, we report five new cases with adenocarinoma ex goblet cell carcinoid. All five patients in our report were initially presented with symptoms of or mimicking appendicitis, with two patients having a perforated appendix, and two patients having diverticulitis. The initial presentation symptoms were RLQ pain, peritonitis from a perforated appendix, and hematochezia. Four of the five patients were male, while one was female with a tumor metastasis to her left ovary four years after her right hemicolectomy.

GCC has a wide range of clinical presentations, therefore, it is important to always consider GCC in patients presenting with abdominal symptoms. Payam S Pahlavan and Rani Kathan reported that the most common clinical presentations for GCC of the appendix are in the order of frequency as follows: acute appendicitis (22.5\%), asymptomatic (5.4\%), non-localized abdominal pain (5.15\%), and abdominal mass (3.09\%) [12]. In female patients, GCC may be presented as Krukenberg tumors, while in half of the female patients it is initially presented as an ovarian mass [13-15]. Although GCC is almost exclusive to the appendix, the extra-appendiceal GCC is exceedingly rare [16]. The only potential risk factor for GCC that has been suggested to date is schitosomiasis [17]. Two of our patients had focally localized tumors. Combining that with the presence of diverticulitis in some of these patients, it may mislead the diagnosis. Therefore, a thorough gross examination of the appendectomy specimen and careful sampling are imperative to the diagnosis of GCC because of the lack of the discrete mass.

The "tang classification" of GCC patients are divided into three groups (A, B, and $\mathrm{C}$ ) and have shown to be a significant prognostic factor $[5,18,19]$. Typical GCC (Group A) was defined as well-defined goblet cells arranged in clusters or in a cohesive linear pattern, with minimal cytologic atypia and architectural distortion of the appendiceal wall, and minimal to no desmoplasia [5]. Adenocarcinoma ex GCC, signet ring cell type (Group B) was defined as goblet cells or signet ring cells arranged in irregular large clusters, with the lack of confluent sheets of cells in a discohesive single file or single cell infiltrating pattern with significant cytologic atypia, and desmoplasia and associated destruction of the appendiceal wall [5]. Adenocarcinoma ex GCC, poorly differentiated carcinoma type (Group C) was defined with the least focal evidence of goblet cell morphology and a component ( $>1$ low power field or $1 \mathrm{~mm}^{2}$ ) that is not otherwise distinguishable from a poorly differentiated adenocarcinoma. It may appear as either gland forming, confluent sheets of signet ring cells, or undifferentiated carcinoma [5]. The 5year survival rate was $100 \%$ in the typical GCC group (Group A), 36\% in Adenocarcinoma ex GCC, signet ring cell type group (Group B), and $0 \%$ in Adenocarcinoma ex GCC, poorly differentiated adenocarcinoma type (Group C). In this study, the five cases include one in Group A, one in Group B, and three in Group C.

An alternative histologic grading system, the simplified two-tier histologic grading system, was proposed, and has also shown good predictive values for the GCC outcome [19]. This histologic scoring system was created whereby one point was given for the presence of each of cytologic atypia, peritumoral stormal desmoplasia, and solid growth pattern (score ranges from 0 to 3 ). A histologic score of $0-1 / 3$ was defined as low grade, and 2-3/3 was defined as high grade. This two-tier grading system demonstrated that the overall 10 -year survival rate in the low-grade histology group was $80.5 \%$, and $0 \%$ in the highgrade histology group. However, the role of $\mathrm{Ki}-67$ as a prognostic marker has been controversial $[5,18,20-23]$. The Ki-67 proliferative index has shown close association with the Tang histologic classification. The Ki-67 staining was relatively low in Group A (11\%) and B (18\%) tumors, and Group C (80\%) tumors demonstrated a high proliferative rate [5]. However, more recent study has shown no correlation between the Ki-67 and the behavior of the GCC tumors [23].

The treatment of GCC still lacks a unanimous consensus. However, it is acknowledged that the complete removal of the GCC in the appendix is imperative, even though the benefit of hemicolectomy in patients with GCC is not clear [24-26]. In addition, the possible bilateral oophorectomy in the female patients is also suggested due to the high incidences of GCC metastasis to the ovaries [13,27]. 


\section{Conclusion}

We describe five new cases with adenocarcinoma ex goblet cell carcinoid, who were presented with symptoms of or mimicking acute appendicitis. The GCC has a wide range of clinical presentations, and more importantly, has no discrete mass except a focally thickened appendiceal wall, and cytopathology examination may reveal no evidence of malignancy. Furthermore, there is a significant overall survival rate difference in patients with the lower grade/stage GCC and higher grade/stage GCC. Therefore, it is important to keep in mind the pitfalls in diagnosis of GCC by careful sampling, so that the patients with this entity can receive the treatment as early as possible for the better outcome.

\section{References}

1. Collins DC (1963) 7,000 Human Appendix Specimens. A Final Report, Summarizing Forty Years' Study. Am J Proctol 14: 265-281.

2. Connor SJ, Hanna GB, Frizelle FA (1998) Appendiceal tumors: retrospective clinicopathologic analysis of appendiceal tumors from 7,970 appendectomies. Dis Colon Rectum 41: 75-80.

3. Subbuswamy SG, Gibbs NM, Ross CF, Morson BC (1974) Goblet cell carcinoid of the appendix. Cancer 34: 338-344.

4. Gallegos NC, Milroy C, Linehan IP, Boulos PB (1992) Crypt cell carcinoma of the appendix. Eur J Surg Oncol 18: 386-387.

5. Tang LH, Shia J, Soslow RA, Dhall D, Wong WD, et al. (2008) Pathologic classification and clinical behavior of the spectrum of goblet cell carcinoid tumors of the appendix. Am J Surg Pathol 32: 1429-1443.

6. Wang HL, Dhall D (2009) Goblet or signet ring cells: that is the question. Adv Anat Pathol 16: 247-254.

7. Burke A, Sobin L (1992) The histogenesis of appendiceal carcinoid tumours. Histopathology 21: 600-601.

8. Burke AP, Sobin LH, Federspiel BH, Shekitka KM, Helwig EB (1990) Goblet cell carcinoids and related tumors of the vermiform appendix. Am J Clin Pathol 94: 27-35.

9. Goddard MJ, Lonsdale RN (1992) The histogenesis of appendiceal carcinoid tumours. Histopathology 20: 345-349.

10. Isaacson P (1981) Crypt cell carcinoma of the appendix (so-called adenocarcinoid tumor). Am J Surg Pathol 5: 213-224.

11. McCusker ME, Coté TR, Clegg LX, Sobin LH (2002) Primary malignant neoplasms of the appendix: a population-based study from the surveillance, epidemiology and end-results program, 1973-1998. Cancer 94: 3307-3312.

12. Pahlavan PS, Kanthan R (2005) Goblet cell carcinoid of the appendix. World J Surg Oncol 3:36.
13. Mandai M, Konishi I, Tsuruta Y, Suginami N, Kusakari T, et al. (2001) Krukenberg tumor from an occult appendiceal adenocarcinoid: a case report and review of the literature. Eur J Obstet Gynecol Reprod Biol 97: 90-95.

14. Baker PM, Oliva E, Young RH, Talerman A, Scully RE (2001) Ovarian mucinous carcinoids including some with a carcinomatous component: a report of 17 cases. Am J Surg Pathol 25: 557-568.

15. Hristov AC, Young RH, Vang R, Yemelyanova AV, Seidman JD, et al. (2007) Ovarian metastases of appendiceal tumors with goblet cell carcinoidlike and signet ring cell patterns: a report of 30 cases. Am J Surg Pathol 31: 1502-1511.

16. Gui X, Qin L, Gao ZH, Falck V, Harpaz N (2011) Goblet cell carcinoids at extraappendiceal locations of gastrointestinal tract: an underrecognized diagnostic pitfall. J Surg Oncol 103: 790-795.

17. Jiang Y, Long H, Li T, Wang W, Liu H, et al. (2012) Schistosomiasis may contribute to goblet cell carcinoid of the appendix. J Parasitol 98: 565-568.

18. Olsen IH, Holt N, Langer SW, Hasselby JP, GrÃ nb $\tilde{A}_{\mathrm{k}} \mathrm{k}$ H, et al. (2015) Goblet cell carcinoids: characteristics of a Danish cohort of 83 patients. PLoS One 10: e0117627.

19. Lee LH, McConnell YJ, Tsang E, Zerhouni S, Speers C, et al. (2015) Simplified 2-tier histologic grading system accurately predicts outcomes in goblet cell carcinoid of the appendix. Hum Pathol 46: 1881-1889.

20. Jiang Y, Long H, Wang W, Liu H, Tang Y, et al. (2011) Clinicopathological features and immunoexpression profiles of goblet cell carcinoid and typical carcinoid of the appendix. Pathol Oncol Res 17: 127-132.

21. Toumpanakis C, Standish RA, Baishnab E, Winslet MC, Caplin ME (2007) Goblet cell carcinoid tumors (adenocarcinoid) of the appendix. Dis Colon Rectum 50: 315-322.

22. Alsaad KO, Serra S, Schmitt A, Perren A, Chetty R (2007) Cytokeratins 7 and 20 immunoexpression profile in goblet cell and classical carcinoids of appendix. Endocrine Pathology 18: 16-22.

23. Liu E, Telem DA, Warner RR, Dikman A, Divino CM (2011) The role of $\mathrm{Ki}-67$ in predicting biological behavior of goblet cell carcinoid tumor in appendix. Am J Surg 202: 400-403.

24. Pham TH, Wolff B, Abraham SC, Drelichman E (2006) Surgical and chemotherapy treatment outcomes of goblet cell carcinoid: a tertiary cancer center experience. Ann Surg Oncol 13: 370-376.

25. Park K, Blessing K, Kerr K, Chetty U, Gilmour H (1990) Goblet cell carcinoid of the appendix. Gut 31:322-324.

26. Goede AC, Caplin ME, Winslet MC (2003) Carcinoid tumour of the appendix. Br J Surg 90: 1317-1322.

27. Tjalma WA, Schatteman E, Goovaerts G, Verkinderen L, Van-den Borre F, et al. (2000) Adenocarcinoid of the appendix presenting as a disseminated ovarian carcinoma: report of a case. Surg Today 30: 78-81. 